\title{
Caractérisation physico-chimique et évaluation de l'activité antiradicalaire des extraits de sept morphotypes de gombo (Abelmoschus spp.) cultivés au Bénin
}

\author{
Rose Estelle KANFON ${ }^{1}$, Marcelline GNAWE ${ }^{2}$, \\ Cokou Pascal AGBANGNAN DOSSA ${ }^{1 *}$, Hounnankpon YEDOMONHAN ${ }^{2}$, \\ D. Valentin WOTTO $^{3}$ et C. K. Dominique SOHOUNHLOUE ${ }^{1}$
}

\author{
${ }^{l}$ Laboratoire d'Étude et de Recherche en Chimie Appliquée (LERCA); Ecole Polytechnique \\ d'Abomey-Calavi / Université d'Abomey-Calavi, République du Bénin. \\ ${ }^{2}$ Laboratoire de Botanique et Écologie Végétale, Faculté des Sciences et Techniques, Université \\ d'Abomey-Calavi, République du Bénin. \\ ${ }^{3}$ Laboratoire de Chimie Physique, Faculté des Sciences et Techniques, Université d'Abomey-Calavi, \\ République du Bénin. \\ *Auteur correspondant, E-mail: cokou2010@gmail.com,cokou2005@yahoo.fr; Tel: +22997078479
}

\section{REMERCIEMENTS}

Les auteurs remercient la Fondation Internationale pour la Science (IFS) pour le financement $n^{\circ}$ C5595-1 qui a couvert les frais de terrain pour la collecte des échantillons de gombo sur tout le territoire du Bénin utilisés dans le présent travail.

\section{RESUME}

Le gombo, de son nom scientifique Abelmoschus spp., est un légume fruit très répandu sur le territoire béninois et consommé par les différents groupes sociolinguistiques qui s'y trouvent. L'objectif de cette étude était de déterminer les propriétés physico-chimiques de sept morphotypes de gombo. Après le screening phytochimique, les composés phénoliques des fruits de sept morphotypes de gombo (Abelmoschus spp.) ont été quantifiés au spectrophotomètre UV-Visible. L'activité antiradicalaire des extraits éthanoliques des fruits a été déterminée à température ambiante par la méthode basée sur la réactivité des extraits avec un radical libre, stable en solution, le 1, 1-DiPhényl-2-PicrylHydrazyle (DPPH). Trois composés de référence, reconnus pour leur pouvoir antioxydant ont servi de référence afin de valider le potentiel antioxydant des extraits étudiés. L'exploration du potentiel phytochimique réalisé sur les sept (07) morphotypes de gombo investiguées a révélé la présence, dans des fruits, des tanins (galliques et catéchiques), des flavonoïdes libres, des sucres réducteurs, des anthraquinones combinés, des mucilages et des leucoanthocyanes tandis que les anthocyanes, les alcaloïdes et les coumarines n'ont été détectés dans aucune des sept morphotypes. Le dosage colorimétrique a montré que la plus forte teneur en composés phénoliques totaux $(2,36 \pm 0,02) \mathrm{mg} / \mathrm{g}$ s'est révélé au niveau du morphotype M52. Les tanins condensés sont plus concentrés dans l'extrait du morphotype M3 $(33,25 \pm 0,89)$ $\mathrm{mg} / \mathrm{g}$. Le morphotype M3 possède la plus forte teneur en flavonoïdes totaux $(10,29 \pm 1,81) \mathrm{mg} / \mathrm{g}$. Les résultats de l'étude de l'activité antiradicalaire (test au DPPH) ont révélé que l'extrait du morphotype M52 est plus actif que les autres morphotypes bien qu'ayant une activité plus faible que celle des standard utilisés. Le gombo constitue donc une source potentielle de composés phénoliques, biomolécules à multiple activités biologiques, qui pourrait être conseillé aussi bien dans les préparations alimentaires que pharmaceutiques. (c) 2018 International Formulae Group. All rights reserved.

Mots clés: Gombo, potentiel phytochimique, activité antiradicalaire. 


\title{
Physico-chemical characterization and antiradical activity of extracts of seven morphotype of okra (Abelmoschus spp.) grown in Benin
}

\begin{abstract}
The Okra, scientifically named Abelmoschus spp., is a fruit vegetable very widespread in Benin and consumed by different sociolinguistic groups of the country. The objective of this study is to evaluate the physico-chemical properties of seven local varieties of okra. After phytochemical screening, the phenolic compounds of the fruits of seven local varieties of okra (Abelmoschus spp) were quantified using a UV-Visible spectrophotometer. The anti-radical activity of the ethanolic extracts of the fruits was determined at room temperature by the method based on the reactivity of the extracts with a free radical, stable in solution, 1.1Diphenyl-2-PicrylHydrazyl (DPPH). Three reference compounds, known for their antioxidant power, served as a reference in order to validate the antioxidant potential of the extracts studied. The exploration of the phytochemical potential carried out on the seven (07) okra varieties investigated revealed the presence in the fruits of tannins (gallic and catechic), free flavonoids, reducing sugars, combined anthraquinones, mucilages and leucoanthocyanins while that anthocyanins, alkaloids and coumarins were not detected in any of the seven varieties. The colorimetric determination shows that the V4 variety with the highest total phenolic content $(2.36 \pm 0.02) \mathrm{mg}$. The condensed tannins are more concentrated in variety V5 $(33.25 \pm 0.89) \mathrm{mg} / \mathrm{g}$. Variety V5 has the highest total flavonoid content $(10.29 \pm 1.81) \mathrm{mg}$. The results of the study of antiradical activity (DPPH test) reveal that the extract of the V4 variety is more active than the other varieties although having a lower activity than the standards used. Okra is therefore a potential source of phenolic compounds, biomolecules with multiple biological activities, which could be recommended in both food and pharmaceutical preparations.
\end{abstract}

(C) 2018 International Formulae Group. All rights reserved.

Keywords: Okra, phytochemical potential, antiradical activity.

\section{INTRODUCTION}

L'être humain s'est toujours appuyé sur la nature pour subvenir à ses besoins de base dont l'alimentation. Celle-ci est de plus en plus variée et joue plusieurs rôles tant sur le plan nutritionnel que sanitaire. En effet, elle doit, au quotidien, répondre aux besoins nutritionnels de l'organisme humain et participer à sa protection contre les agressions externes, notamment les maladies et le vieillissement précoce (Gbohaïda et al., 2016). Mais si l'on insiste sur les mets à éviter, on oublie souvent de parler de ceux à privilégier : les fruits et légumes dont le gombo. Ceux-ci sont riches en de nombreuses molécules biologiquement actives (nutriments, antioxydants) (Itoua et al., 2015). L'ingestion des antioxydants tels que les composés phénoliques, par l'intermédiaire des fruits et des légumes, pourrait permettre à notre organisme de renforcer ses moyens de défense contre les processus d'oxydation qui menacent quotidiennement nos cellules (Khan, 2010). Ces composés ont la capacité de piéger les radicaux libres qui sont générés en permanence par notre organisme ou formés en réaction à des agressions de notre environnement (tabac, polluants, infections,...) (Gbohaïda et al., 2015). De la famille des malvacées, le gombo est une plante tropicale à fleurs, originaire d'Afrique qui possède de nombreuses vertus pour l'homme. Il est souvent considéré comme l'aliment des pauvres, tel que l'indique la dénomination que lui attribuent les Antillais, « asperge du pauvre ». Mais, c'est sans connaître les vertus médicinales insoupçonnées dont la nature providence a pourvu ce légume de couleur vert foncé à la peau ferme et recouverte d'un duvet soyeux. C'est une plante exceptionnelle et originale car toutes ses parties (racines, tiges, feuilles, fruits, graines) sont valorisées sur les plans alimentaire, médicinal, artisanal et même industriel (Ouedraogo, 2009). Mangé cru, cuit ou parfois sous forme déshydratée, le gombo est un légume qui procure un apport intéressant en antioxydants et est très 
consommé sur tous les tropiques. Malgré les vertus de la plante, très peu de données ont été rapportées dans la littérature concernant sa composition chimique, sa teneur en composés phénoliques et sur les activités biologiques de ses extraits. Il reste alors mal connu du monde scientifique, d'où l'intérêt de la présente étude. L'objectif principal de ce travail était de déterminer les caractéristiques physico-chimiques des fruits de sept morphotypes de gombo à travers l'identification des métabolites secondaires, l'évaluation de leurs teneurs en composés phénoliques ainsi que l'activité antioxydante de leurs extraits.

\section{MATERIEL ET METHODES \\ Matériel végétal}

Il est constitué des fruits prématures du gombo. L'étude a porté sur sept (07) morphotypes des fruits du gombo $\left(\mathrm{M}_{43}, \mathrm{M}_{4}\right.$, M29, M52, M3, M41, M23) obtenus par sélection variétale parmi plusieurs cultivars collectés à travers le Bénin et plantés à la ferme expérimentale du Département de Botanique et Écologie Végétale de la Faculté des Sciences et Techniques de l'Université d'Abomey-Calavi (FAST/ UAC). Elles diffèrent entre elles par l'aspect général de la plante, la forme, la couleur des feuilles et des fruits.

\section{Matériel de laboratoire}

$\mathrm{Au}$ laboratoire, les réactifs chimiques (réactif de Folin - Ciocalteu, liqueur de Fehling...), les solvants organiques (éthanol, chloroforme...) et les appareils (lampe UV, rotavapor, étuve, spectrophotomètre...) ont été utilisés.

\section{Collecte et traitement des échantillons}

Les fruits de gombo ont été collectés à l'Université d'Abomey- Calavi (UAC/Bénin). Après collecte, ils ont été soigneusement lavés avec de l'eau de robinet puis nettoyés avec du papier absorbant. Découpés en petits morceaux, ils ont été séchés ensuite à l'étuve à $35{ }^{\circ} \mathrm{C}$ jusqu'à stabilisation de leur masse pour éviter l'oxydation des composés phénoliques puis réduits en poudre à l'aide d'un broyeur. La poudre récupérée a été conservée dans une bouteille pour les analyses.

\section{Caractérisation physico-chimique des fruits de sept morphotypes de gombo \\ Screening phytochimique}

$\mathrm{La}$ première étape d'une étude phytochimique est la recherche des grandes classes de composés chimiques de la plante à étudier. En effet, le criblage phytochimique consiste à réaliser une analyse chimique qualitative basée sur des réactions de coloration ou de précipitation plus ou moins spécifiques à chaque classe de principes actifs. Les groupes phytochimiques recherchés sont, entre autres, les alcaloïdes, les composés phénoliques (flavonoïdes, anthocyanes, tanins,...), les saponosides, les coumarines, les mucilages, les sucres réducteurs (osesholosides).

- Flavonoïdes: l'identification des flavonoïdes a été réalisée par le test à la cyanidine (Agbangnan et al., 2013).

- Tanins: ils ont été mis en exergue par le test de Stiasny (Soro, 2009).

- Saponosides: ils ont été déterminés par le test de mousse; degré de dilution d'un décocté aqueux donnant une mousse persistante après agitation (Dohou, 2003).

- Composés phénoliques: la détermination des composés appartenant à ce groupe a été faite par la réaction au chlorure ferrique (Agbangnan et al., 2013).

- Alcaloïdes : ils ont été identifiés par le test de Meyer et confirmés par le test de Bouchardat (N'Guessan et al., 2009).

- Anthraquinones : ils ont été identifiés par le test de Borntrager (Dohou, 2003)

- Mucilages: l'obtention d'un précipité floconneux d'un décocté dans l'éther éthylique indique la présence des mucilages (Traoré, 2010).

- Coumarines: les coumarines ont été mises en évidence par la fluorescence à l'UV à 365 nm (Soro ; 2009).

\section{Préparation des extraits éthanoliques}

Pour l'analyse quantitative des composés phénoliques, l'extraction liquidesolide a été réalisée par macération dans 
l'éthanol $\left(70^{\circ}\right)$ avec un ratio de $10 \% .10 \mathrm{~g}$ de poudre végétale ont été pesés puis mélangés avec un volume de $100 \mathrm{~mL}$ du solvant. Le mélange est maintenu sous agitation magnétique pendant 24 heures à température ambiante. La solution obtenue est ensuite filtrée sur papier filtre (Wattman $\mathrm{N}^{\circ} 1$ de diamètre $0,16 \mathrm{~mm}$ ) sous vide. Le filtrat a été ensuite récupéré et l'opération a été répétée 2 fois (soit 72 heures d'extraction au total) mais avec $50 \mathrm{~mL}$ du solvant dès le deuxième jour. Le volume total du filtrat est concentré sous vide à $60{ }^{\circ} \mathrm{C}$ à l'aide d'un rotavapor de type Heidolph. L'extrait sec a été ensuite récupéré, pesé, étiqueté et conservé à $4{ }^{\circ} \mathrm{C}$ jusqu'à l'utilisation. Le rendement (R) d'extraction définis comme étant le rapport entre la masse obtenue de l'extrait sec et la masse du matériel végétal traité a été calculé par la formule suivante :

$$
\boldsymbol{R}(\%)=(\boldsymbol{M e} / \mathbf{M v}) \times \mathbf{1 0 0}
$$

$\boldsymbol{R}(\%)$ : Rendement en \%; $\boldsymbol{M e}$ : Masse de l'extrait après l'évaporation du solvant et $\boldsymbol{M v}$ : Masse de la matière végétale utilisée pour l'extraction.

\section{Dosage des composés phénoliques}

La méthode colorimétrique par spectrophotométrie a été utilisée pour la quantification des composés phénoliques à partir de la matière végétale.

- Composés phénoliques totaux: Pour ce dosage, $0,4 \mathrm{~mL}$ de l'extrait des fruits est introduit dans un tube à essai et $2 \mathrm{~mL}$ du réactif de Folin-Ciocalteu (1N) sont ajoutés puis 4 minutes après, il est ajouté $1,6 \mathrm{~mL}$ d'une solution de $\mathrm{Na}_{2} \mathrm{CO}_{3}$ (7\%). Le mélange obtenu est incubé à l'abri de la lumière et à la température ambiante pendant 2 heures. L'absorbance est ensuite mesurée au spectrophotomètre à $765 \mathrm{~nm}$ contre un blanc. Notons qu'une droite d'étalonnage est préalablement réalisée avec l'acide gallique dans les mêmes conditions que les échantillons à analyser. Les résultats obtenus sont exprimés en milligramme équivalent $\mathrm{d}$ 'acide gallique par gramme de matière sèche (mg EAG/g Ms).

- Flavonoïdes totaux: Les flavonoïdes totaux ont été quantifiés par la méthode du trichlorure d'aluminium $\left(\mathrm{AlCl}_{3}\right)$. Cette technique est basée sur la formation du complexe brunâtre flavonoïde-aluminium qui a une absorption maximale à $510 \mathrm{~nm}$ (Enujiugha, 2010; Agbangnan et al., 2012). Les résultats sont exprimés en milligramme équivalent de quercétine par gramme de matière sèche (mg EQ/g Ms). Une courbe d'étalonnage est élaborée avec des solutions standard de quercétine préparées à différentes concentrations.

- Tanins condensés: Le dosage des tanins condensés a été réalisé par la méthode à la vanilline sulfurique (Agbangnan et al., 2012). Le principe de ce dosage est basé sur la fixation du groupe aldéhydique de la vanilline sur le carbone en position 6 du cycle A des tanins condensés qui entraînent une coloration verte de la solution qui absorbe à $500 \mathrm{~nm}$. Les résultats sont exprimés en milligramme équivalent catéchine par gramme de matière sèche $\left(\begin{array}{lll}m g & E C / g & M s\end{array}\right)$. Une courbe d'étalonnage est élaborée avec des solutions standard de catéchine préparées à différentes concentrations.

\section{Détermination de l'activité antiradicalaire} des extraits des fruits de gombo

La méthode d'évaluation de l'activité antiradicalaire des extraits non volatils est inspirée de celle décrite dans la littérature. Le principe de cette méthode est basé sur la mesure du pourcentage de piégeage des radicaux libres d'une solution de DPPH. Ce piégeage est visualisé par la disparition de la couleur pourpre du DPPH. Les cuves sont laissées à l'obscurité pendant une heure et les absorbances ont été mesurées à $517 \mathrm{~nm}$ (Brand-Willams, 1995; Agbangnan et al., 2013; Gisèle et al., 2018). Le Pourcentage d'Inhibition (de piégeage de radicaux libres DPPH) est calculé par la formule :

$$
\mathbf{P I}=\left[\left(\mathbf{A}_{\mathrm{bl}}-\mathbf{A}_{\mathrm{ext}}\right) / \mathbf{A}_{\mathrm{bl}}\right] \mathbf{x 1 0 0}
$$

PI : Pourcentage d'Inhibition ou pourcentage de piégeage ;

$\mathbf{A}_{\mathbf{b l}}$ : Absorbance du blanc (ne contenant aucun antioxydant) et $\mathbf{A}_{\text {ext }}$ : Absorbance des extraits Entre les différentes concentrations et les PI s'établit une régression linéaire à partir de laquelle est déduite la valeur correspondante à la $\mathrm{CI}_{50}$ (Concentration de l'antioxydant pour laquelle 50\% des radicaux libres sont inhibés). 


\section{RESULTATS}

\section{Screening phytochimique}

Le criblage phytochimique a permis de mettre en évidence plusieurs métabolites au niveau des fruits du gombo (Tableau 1). On y note la présence des composés chimiques tels que les tanins (galliques et catéchiques), les flavonoïdes libres et les composés réducteurs. Les saponosides ont été détectés dans les morphotypes M43, M52 et M23 (car révélés par un indice de mousse supérieur à 100) alors que ces derniers ont été absents dans les morphotypes M4, M29, M3 et M41. On a également noté la présence des leucoanthocyanes et des mucilages dans les morphotypes M29, M52, M3, M23. Ces derniers n'ont pas été observés au niveau de la morphotype M43 alors que la morphotype M4 contient des mucilages mais ne contient pas de leucoanthocyanes. Les coumarines sont présents dans les morphotypes M52 et M23 mais absents dans les morphotypes M43, M4, M29, M3 et M41. Les composés de type anthraquinones (surtout les anthraquinones libres) sont uniquement présents dans les morphotypes M43 et M4.

\section{Rendement d'extraction et propriétés physiques des extraits}

Le Tableau 2 présente les rendements d'extraction du matériel végétal étudié et les propriétés physiques de ses extraits aqueux et éthanolique.

\section{Dosage des composés polyphénoliques Teneur en Composés Phénoliques Totaux (CPT)}

La courbe d'étalonnage établie donne une droite d'équation: $\mathrm{y}=8,5927 \mathrm{x}-0,006$ avec un coefficient de détermination $\mathrm{R}^{2}=$ 0,9853 (Figure 2). La teneur en composés phénoliques exprimée en $\mathrm{mg}$ équivalent d'acide gallique par $g$ de matière sèche varie très peu dans les différents échantillons de gombo investigués (Figure 3). La plus forte teneur en composés phénoliques totaux a été notée au niveau des fruits du morphotype M52 $(2,36 \mathrm{mg} / \mathrm{g})$. Les morphotypes M43 $(2,14 \mathrm{mg} / \mathrm{g}) ;$ M41 $(2,10 \mathrm{mg} / \mathrm{g}) ;$ M3 (2,07 $\mathrm{mg} / \mathrm{g})$ et M29 $(2,03 \mathrm{mg} / \mathrm{g})$ présentent une teneur moyenne alors que M4 $(1,76 \mathrm{mg} / \mathrm{g})$ et M23 (1,55 mg/g) sont les deux morphotypes montrant des teneurs relativement faibles en ces composés.

\section{Teneur en flavonoüdes totaux (FVT)}

Le composé de référence utilisé pour l'établissement de la courbe d'étalonnage est la quercétine. Elle donne une droite d'équation $\mathrm{y}=0,6822 \mathrm{x}+0,0079$ avec un coefficient de détermination $\mathrm{R}^{2}=0,988$ (Figure 4). Les résultats obtenus sont exprimés en $\mathrm{mg}$ équivalent quercétine par gramme de matière sèche $(\mathrm{mg} \mathrm{EC} / \mathrm{g})$. La Figure 5 traduisant la teneur en flavonoïdes totaux des différents morphotypes étudiées a montré que la plus forte teneur en flavonoïdes totaux a été observée au niveau du morphotype M3 (10,29 $\mathrm{mg} / \mathrm{g})$; viennent ensuite les morphotypes M43 (7,27 mg/g) et M4 (7,04mg/g). Les morphotypes M52 (6,26 $\mathrm{mg} / \mathrm{g}) ; \mathrm{M} 41(6,12 \mathrm{mg} / \mathrm{g}) ; \mathrm{M} 29(5,96 \mathrm{mg} / \mathrm{g})$ et M23 (5,81 $\mathrm{mg} / \mathrm{g})$ ont des teneurs en flavonoïdes totaux très proches.

\section{Teneur en Tanins Condensés (TC)}

Une courbe d'étalonnage établit à partir des solutions de différentes concentrations de catéchine et représentée par une droite d'équation $\mathrm{y}=0,626 \mathrm{x}-0,0118$ avec un coefficient de détermination $\mathrm{R}^{2}=$ 0,9907 (Figure 6) a permis d'exprimer la concentration en tanins condensés des échantillons investigués. La Figure 7 laisse observer une forte teneur en tanins condensés au niveau des morphotypes M3 (33,29 $\mathrm{mg} / \mathrm{g}) ;$ M52 $(32,33 \mathrm{mg} / \mathrm{g})$ et M43 (30,03 $\mathrm{mg} / \mathrm{g}$ ). Viennent ensuite les morphotypes M29 $(24,69 \mathrm{mg} / \mathrm{g})$ et M41 $(22,44 \mathrm{mg} / \mathrm{g})$ présentant une teneur en tanins condensés relativement élevée. Enfin les morphotypes M23 (19,98 $\mathrm{mg} / \mathrm{g})$ et M4 $(19,74 \mathrm{mg} / \mathrm{g})$ ont présenté les faibles teneurs en tanins condensés.

\section{Evaluation de l'activité antiradicalaire des extraits éthanoliques}

Pour évaluer l'activité antiradicalaire de nos extraits de gombo, trois composés de référence reconnus pour leur pouvoir antiradicalaire ont été utilisés : Acide Gallique (AG), ButylHydroxyAnizole (BHA) et la Quercétine $(\mathrm{Q})$. Les résultats exprimés en pourcentage de piégeage de radicaux libres 
DPPH en fonction de la concentration des extraits des échantillons ont permis d'obtenir la concentration de l'extrait pour laquelle 50\% de radicaux libres de DPPH sont piégés $\left(\mathrm{CI}_{50}\right)$. Ces résultats sont reportés dans le Tableau 3. Il ressort de l'analyse de ce tableau que la $\mathrm{CI}_{50}$ des standards utilisés varie de 0,03 à 0,04 $\mathrm{mg} / \mathrm{mL}$. La quercétine a présenté la plus faible $\mathrm{CI}_{50}$ donc possède la plus forte activité antiradicalaire. Elle est suivie du BHA et de l'Acide Gallique. Signalons que plus actif est un composé, plus faible est sa $\mathrm{CI}_{50}$. Les $\mathrm{CI}_{50}$ des extraits analysés ont varié de 9,9 à 85 $\mathrm{mg} / \mathrm{mL}$. Toutes les morphotypes de gombo étudiées ont présenté une $\mathrm{CI}_{50}$ plus élevée que celle des trois standard utilisés et donc une activité antiradicalaire plus faible. $\mathrm{La} \mathrm{CI}_{50}$ la plus faible a été obtenue avec l'extrait du morphotype M52 (9,9 mg/mL) de gombo; ce qui révèle donc l'activité antiradicalaire la plus élevée, suivie des extraits des morphotypes M29 (12,5 mg/mL) et M43 (25 $\mathrm{mg} / \mathrm{mL}$ ). L'extrait du morphotype M23 (85 $\mathrm{mg} / \mathrm{mL}$ ) a montré la valeur la plus élevée de la $\mathrm{CI}_{50}$ donc l'activité antiradicalaire la plus faible. Viennent ensuite les extraits des morphotypes M4 $(60,05 \mathrm{mg} / \mathrm{mL})$ et $\mathrm{M} 3$ $(56,5 \mathrm{mg} / \mathrm{mL})$. Le morphotype M41 a une $\mathrm{CI}_{50}$ moyenne $(47 \mathrm{mg} / \mathrm{mL})$, par conséquent une activité antiradicalaire moyenne.

Tableau 1: Screening phytochimique des fruits de Abelmoschus esculentus.

\begin{tabular}{|c|c|c|c|c|c|c|c|c|}
\hline \multirow{2}{*}{\multicolumn{2}{|c|}{ Familles de composés recherchés }} & \multicolumn{7}{|c|}{ Echantillons } \\
\hline & & \multirow{2}{*}{$\begin{array}{l}\mathbf{V 1} \\
+\end{array}$} & \multirow{2}{*}{$\begin{array}{l}\mathrm{V2} \\
+\end{array}$} & \multirow{2}{*}{$\begin{array}{l}\mathrm{V3} \\
+\end{array}$} & \multirow{2}{*}{$\begin{array}{l}\mathrm{V4} \\
+\end{array}$} & \multirow{2}{*}{$\begin{array}{l}\text { V5 } \\
+\end{array}$} & \multirow{2}{*}{$\begin{array}{l}\text { V6 } \\
+\end{array}$} & \multirow{2}{*}{$\begin{array}{l}\text { V7 } \\
+\end{array}$} \\
\hline & Tanins totaux & & & & & & & \\
\hline & Tanins catéchiques & + & + & + & + & + & + & + \\
\hline Tanins & Tanins galliques & + & + & + & + & + & + & + \\
\hline \multirow{3}{*}{ Flavonoïdes } & Anthocyanes & - & - & - & - & - & - & - \\
\hline & Flavonoïdes libres & + & + & + & + & + & + & + \\
\hline & Leucoanthocyanes & - & - & + & + & + & + & + \\
\hline \multicolumn{2}{|l|}{ Mucilage } & - & + & + & + & + & + & + \\
\hline \multicolumn{2}{|l|}{ Alcaloïdes } & - & - & - & - & - & - & - \\
\hline \multicolumn{2}{|c|}{ Sucres réducteurs } & + & + & + & + & + & + & + \\
\hline \multicolumn{2}{|c|}{ Anthraquinones libres } & + & + & - & - & - & - & - \\
\hline \multirow{3}{*}{$\begin{array}{l}\text { Anthraquinones } \\
\text { combinés }\end{array}$} & O-hétérosides & - & + & + & - & - & - & - \\
\hline & $\begin{array}{l}\text { O-hétérosides à } \\
\text { génine réduite }\end{array}$ & + & + & + & + & + & + & - \\
\hline & C-hétérosides & + & + & + & + & + & + & - \\
\hline \multicolumn{2}{|l|}{ Coumarines } & - & - & - & + & - & - & + \\
\hline \multirow{2}{*}{\multicolumn{2}{|c|}{$\begin{array}{l}\text { Saponosides } \\
\text { (Indice de mousse) }\end{array}$}} & + & - & - & + & - & - & + \\
\hline & & 125 & $<100$ & $<100$ & 120 & $<100$ & $<100$ & 175 \\
\hline
\end{tabular}


Tableau 2: Rendement d'extraction et propriétés physiques des extraits.

\begin{tabular}{ccccc}
\hline Morphotypes & $\begin{array}{c}\text { Rendement } \\
\text { éthanolique (\%) }\end{array}$ & Aspect physique & Couleur & Soluble dans \\
\hline M43 & 3,92 & & & \\
M4 & 61,00 & & & \\
M29 & 1,82 & Pourpre & Ethanol \\
M52 & 5,00 & Poudre & & \\
M3 & 4,00 & & & \\
M41 & 13,86 & & & \\
M23 & 8,00 & & & \\
\hline
\end{tabular}

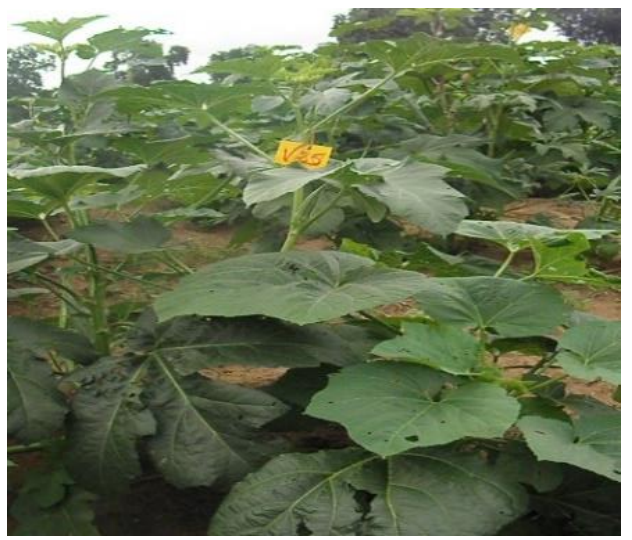

Plante du gombo

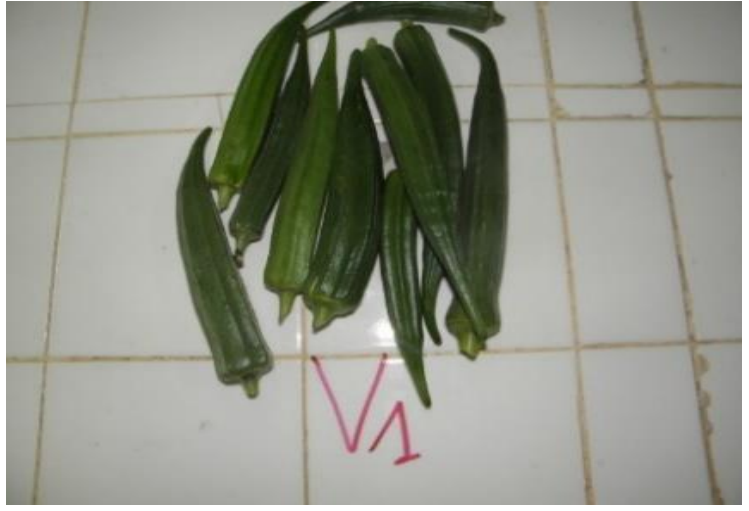

Fruits du gombo

Figure 1 : Photos du gombo (Abelmoschus spp.).

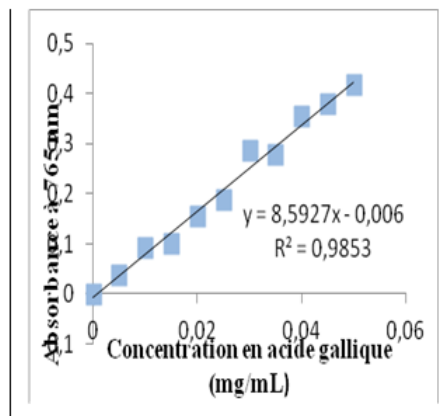

Figure 2: Courbe d'étalonnage de l'acide gallique.

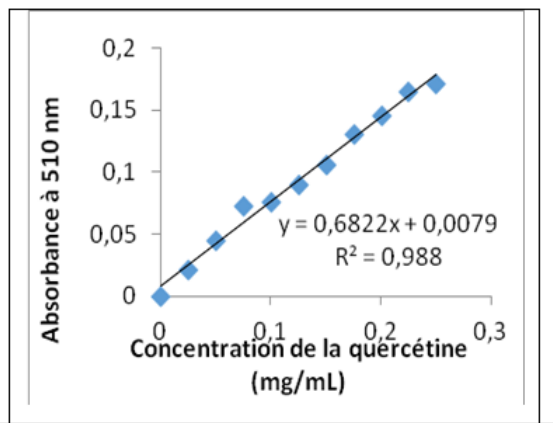

Figure 4: Courbe d'étalonnage de la quercétine.

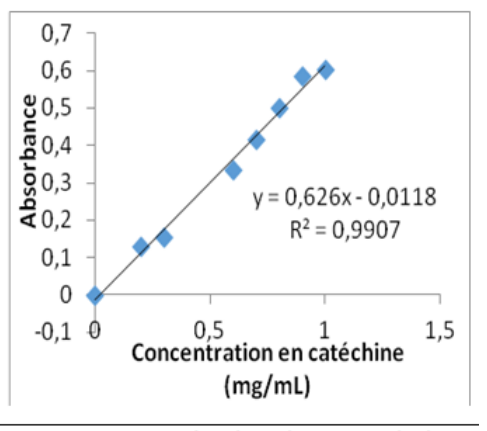

Figure 6: Courbe d'étalonnage de la catéchine. 


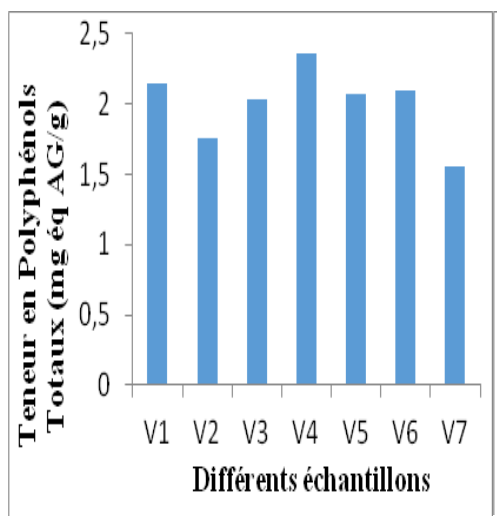

Figure 3: Teneurs en Composés Phénoliques Totaux (PPT) des morphotypes de gombo.

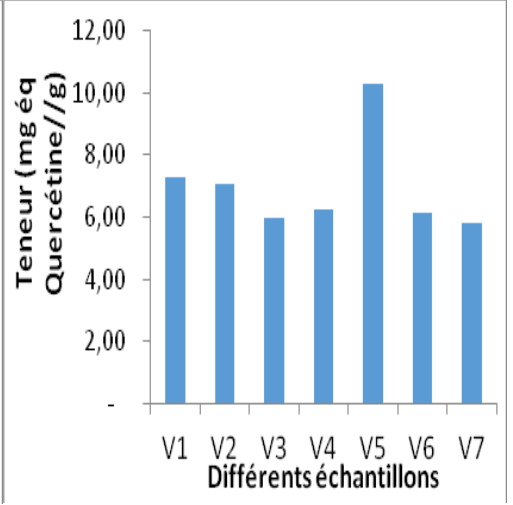

Figure 5 : Teneur en Flavonoïdes Totaux des différents morphotypes étudiés.

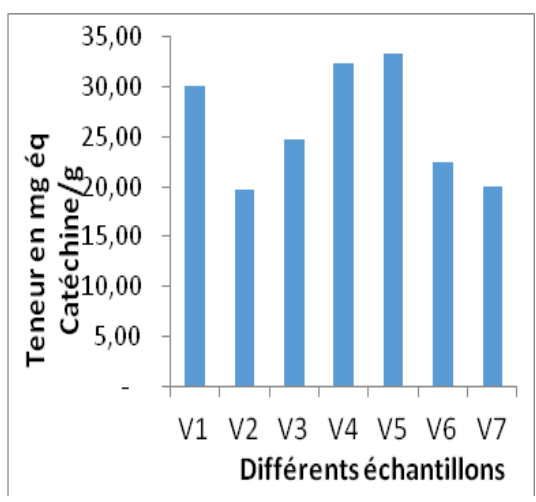

Figure 7: Teneur en Tanins condensés des différents morphotypes.

Tableau 3: Teneur en CPT et $\mathrm{CI}_{50}$ des extraits et des standards.

\begin{tabular}{ccc}
\hline Echantillon & $\mathbf{C I}_{\mathbf{5 0}}(\mathbf{m g} / \mathbf{m L})$ & $\begin{array}{c}\text { Teneur en composés phénoliques totaux } \\
(\mathbf{m g} / \mathbf{g})\end{array}$ \\
\hline V1 & 25,00 & $2,14 \pm 0,03$ \\
\hline V2 & 60,05 & $1,76 \pm 0,12$ \\
\hline V3 & 12,50 & $2,03 \pm 0,03$ \\
\hline V4 & $\mathbf{9 , 9 0}$ & $\mathbf{2 , 3 6 \pm 0 , 0 2}$ \\
\hline V5 & 56,50 & $2,07 \pm 0,04$ \\
\hline V6 & 47,00 & $2,07 \pm 0,03$ \\
\hline V7 & $\mathbf{8 5 , 0 0}$ & $\mathbf{1 , 5 5 \pm 0 , 0 8}$ \\
\hline AG & 0,04 & \\
\hline Q & 0,03 & \\
\hline BHA & 0,04 & \\
\hline & & \\
\hline & & \\
\hline & & \\
\hline & & \\
\hline
\end{tabular}




\section{DISCUSSION}

L'exploration du potentiel phytochimique des différentes morphotypes des fruits de gombo étudiés a révélé qu'elles contiennent toutes des tanins, des flavonoïdes, des sucres réducteurs, des mucilages. La majorité des morphotypes étudiés est pourvue d'anthraquinones et de mucilages mais dépourvue de coumarines et de saponosides (Tableau 1). Ces résultats sont en accord avec ceux des travaux de Shankul (2014) qui ont révélé dans le gombo, entre autres métabolites, la présence des flavonoïdes. Nos résultats confirment également ceux de Liao et al. (2012) sur Abelmoscus esculentus qui ont montré que les différentes parties de ce légume contiennent des composés phénoliques et des flavonoïdes contrairement aux travaux de Kumar et al. (2009) qui ont noté dans les mucilages de gombo la présence des saccharides et l'absence des tanins, des alcaloïdes, des flavonoïdes et des sucres réducteurs. Cette différence observée serait due à la situation géographique et aux conditions climatiques du milieu de récolte ou à l'organe investigué de la plante. Les tanins permettent de stopper les hémorragies et de lutter contre les infections. Les végétaux riches en tanins sont utilisées pour retendre les tissus souples comme dans le cas des veines variqueuses, pour drainer les secrétions excessives, comme dans la diarrhée et pour réparer les tissus endommagés par un eczéma ou une brulure (Boukri, 2014). L'anthraquinone et ses dérivés actifs comme les glucosides d'anthraquinone stimulent le péristaltisme de l'intestin grêle et augmentent les mouvements péristaltiques du côlon. Les glucosides d'anthraquinone se transforment dans le côlon en sennosides. Ces derniers sont hydrophiles et réduisent l'absorption de l'eau en vue d'avoir un bol fécal fluide (Emmanuel, 2008). Ils évitent, par conséquent, la formation de selles grumeleuses. L'anthraquinone est utilisée comme laxatif ou purgatif. Les morphotypes M43, M4, M29, M52, M3 et M41 du gombo peuvent donc être conseillées dans l'alimentation pour faciliter la digestion, combattre la constipation et soigner l'incontinence urinaire. On pourrait donc attribuer à ces morphotypes de gombo la vertu d'apaiser les troubles gastro-intestinaux. Les coumarines possèdent des propriétés très diverses et sont capables de prévenir la peroxydation des lipides membranaires (Boukri, 2014). Les morphotypes M52 et M23 du gombo, riches en ces métabolites, peuvent donc être conseillées dans l'alimentation et entrer dans la composition de divers produits pharmaceutiques et cosmétiques pour soigner une peau irritée ou soulager les ampoules aux pieds et aux mains. L'absence des anthocyanes, puissants antioxydants, peut être due au fait que ces substances sont fragiles et rapidement dégradables. Ceux-ci nettoient l'organisme des radicaux libres et maintiennent une bonne circulation du sang (Agbangnan et al., 2013). Ce légume serait donc pourvu de vertus nutritionnelles et médicinales. Cette étude préliminaire de screening chimique a révélé la présence des composés phénoliques dans les variétés ciblées et a permis de sélectionner la méthode d'extraction de même que les différentes méthodes d'analyses physicochimiques applicables à la quantification de ces métabolites secondaires dans nos échantillons.

$\mathrm{Au}$ vu des résultats obtenus (Tableau 2), il apparaît un grand écart entre le rendement d'extraction éthanolique des morphotypes M4 (61\%), M41 $(13,86 \%)$ et M29 (1,82\%). Toutefois, les valeurs obtenues sont en moyenne en accord avec celles obtenues par Kunli et al. (2018). En effet, il est difficile de comparer les valeurs de nos rendements avec d'autres études, car le rendement est relatif et semble être lié aux propriétés génétiques des plants, à leur origine géographique ainsi qu'au stade des fruits avant récolte et aux conditions d'extraction (Kodjo et al., 2017). Tous les extraits obtenus ont présenté les mêmes caractéristiques physiques. Ils ont un aspect de poudre, de couleur pourpre et sont tous solubles dans l'éthanol.

Les résultats des analyses quantitatives en composés phénoliques par spectrophotométrie UV-visible des extraits des différentes variétés de gombo, exprimés en $\mathrm{mg} / \mathrm{g}$ de matière sèche, ont montré qu'ils sont majoritairement constitués des flavonoïdes. Ils présentent une teneur moyenne en composés phénoliques totaux et une faible teneur en tanins condensés. Nos résultats corroborent ceux de Liao et al. 
(2012) en ce sens que les teneurs en composés phénoliques et en flavonoïdes totaux trouvés par ces auteurs et exprimés respectivement en (mg GAE/g de MS) et (mg RUT/g de MS) varient de $1,9473 \pm 0,0853$ à $324,8386 \pm 4,7324$ pour les premiers et $6,0326 \pm 0,1802$ à $976,0607 \pm 2,6126$ pour les seconds. Adetuyi, et al. en 2014 avait aussi révélé la présence des composés phénoliques dans les graines de gombo. La teneur en phénols des gousses de gombo analysées par ces auteurs variait de 185 mg GAE / $100 \mathrm{~g}$ à 1460 mg GAE / $100 \mathrm{~g}$ d'échantillon. Les travaux de Fangbo et al. (2015) sur l'étude des constituants antioxydants et anti-fatigue du gombo ont montré que la teneur en polyphénols des graines de gombo était d'environ 29,5\% contre $1,25 \%$ pour la peau. Parmi les extraits de gombo analysés dans la présente étude, l'extrait de la variété V4 est celui ayant développé la plus forte activité antiradicalaire. Il est par conséquent le plus antioxydant $\left(\mathrm{CI}_{50}\right.$ $=9,9 \mathrm{mg} / \mathrm{mL}$ ). D'après les travaux de Liao et al. (2012), Kouadio et al. (2017) puis Serigne et al. (2017), les flavonoïdes et les composés phénoliques seraient les principaux composants responsables de l'activité antioxydante des extraits et il existerait une relation directe entre les teneurs en flavonoïdes, en composés phénoliques et l'activité antioxydante. Adelakun et al. (2009) puis Huang et al. (2007) ont montré que les polyphénols et les flavonoïdes des graines de A. esculentus pourraient être les constituants antioxydants et anti-fatigue de ce légume fruit. Nos résultats confirment les observations de ces auteurs car le morphotype M52 qui s'est avérée être la plus riche en composés polyphénoliques totaux s'est aussi révélée la plus antioxydante. Le gombo et particulièrement le morphotype M52 pourrait être conseillée et utilisée grâce à sa propriété anti-oxydante et anti-fatigue pour renforcer le système immunitaire et aider l'organisme à lutter contre les radicaux libres. Les antioxydants sont utilisés pour préserver les aliments de la décoloration, du rancissement et de la détérioration due à l'auto-oxydation (Emmanuel M, 2008). La drogue de gombo pourrait donc être utilisée comme un additif alimentaire. Les extraits ayant exhibé un pouvoir antioxydant intéressant pourraient être suggérés pour leur utilisation en agroalimentaire, en cosmétique et en industrie pharmaceutique.

\section{Conclusion}

La flore africaine riche et diversifiée, regorge de nombreuses plantes utilisées dans l'alimentation et en médecine traditionnelle. Parmi elle, figure le gombo (Abelmoschus spp.) dont les fruits de sept morphotypes (M43, M4, M29, M52, M3, M41 et M23) ont fait l'objet de notre étude qui s'est focalisée sur la détermination des caractères physicochimiques de même que l'évaluation du potentiel antiradicalaire de leurs extraits éthanoliques. Les résultats du screening phytochimique montrent que les fruits des différentes morphotypes de Abelmoschus spp. étudiées contiennent des métabolites secondaires tels que les tanins (galliques et catéchiques), les flavonoïdes libres, les sucres réducteurs, les anthraquinones combinés, les mucilages et les leucoanthocyanes. $\mathrm{La}$ présence de ceux-ci dans nos échantillons serait responsable des propriétés antioxydante, hypoglycémiante, et de l'effet préventif de l'obésité démontrés dans la littérature. Ils constituent donc un moyen de prévention et surtout de lutte contre les maladies cardiovasculaires. Le morphotype M4 a le meilleur rendement d'extraction. La plus forte teneur en composés phénoliques totaux a été obtenue au niveau de l'extrait des fruits du morphotype M43 tandis que celui des fruits du morphotype M3 est le plus riche en flavonoïdes totaux. Les extraits des morphotypes M43, M52 et M3 ont été les plus concentrés en tanins condensés. L'évaluation de l'activité antiradicalaire (test au DPPH) a révélé que les extraits testés ont une activité antiradicalaire plus faible que celle des trois molécules de références. Seuls les extraits des fruits des morphotypes M52 et M29 sont dotés d'un pouvoir antiradicalaire intéressant. Pour une meilleure compréhension du mode d'action des composés phénoliques, il s'avère intéressant après isolement et caractérisation d'évaluer "in vitro" et "in vivo" l'activité antioxydante de chacun de ces composés pris séparément. Au vu des résultats, le gombo est un aliment possédant des vertus nutritionnelles et médicinales pouvant donc être valorisé dans plusieurs domaines. 


\section{CONFLIT D'INTERETS}

Les auteurs déclarent qu'il n'y a aucun conflit d'intérêts en lien avec cet article.

\section{CONTRIBUTIONS DES AUTEURS}

KRE a participé à toutes les phases du travail, de la collecte du matériel végétal à la rédaction du manuscrit en passant par les analyses au laboratoire. GM et YH ont participé à la collecte du matériel végétal sur toute l'étendue du territoire nationale, à la sélection variétale, à la mise en place du champ expérimental puis à la rédaction. $\mathrm{ADCP}$ a participé à la conception de l'étude et à toutes les phases du travail, en l'occurrence les analyses au laboratoire, l'interprétation des données et la rédaction du manuscrit. WDV et SCKD ont supervisé toutes les opérations de manipulation au laboratoire d'interprétation des données et de rédaction du manuscrit.

\section{REMERCIEMENTS}

Les auteurs remercient également les docteurs MINDOATINSA Espérance et GBAHAÏDA Virginie pour leur assistance technique.

\section{REFERENCES}

Adelakun OE, Oyelade OJ, Ade-Omowaye BI, Adeyemi IA, Van de Venter M. 2009. Chemical composition and the antioxidative properties of Nigerian okra seed (Abelmoschus esculentus Moench) flour. J Food Chem Toxicol., 47: 11231126.

Adetuyi F, Ibrahim T. 2014. Effect of Fermentation Time on the Phenolic, Flavonoid and Vitamin C Contents and Antioxidant Activities of Okra (Abelmoschus esculentus) Seeds. NIFOJ , 32(2) : 128 - 137.

Agbangnan DCP, Noudogbessi JP, Chrostowska A, Tachon C, Fouquet E, Sohounhloue C KD. 2013. Phenolic compound of benin's red sorghum and their antioxidant properties. Asian $J$. Pharm Clin. Res., 6(2): 277-280.

Agbangnan PD, Tachon C, Bonin $\mathrm{H}$, Chrostowska A, Fouquet E, Sohounhloue CKD. 2012. Phytochemical study of a tinctorial plant of Benin traditional pharmacopoeia: The red sorghum (sorghum caudatum) of Benin.
Scientific Study \& Research, 13(2): 121135.

Boukri NEH. 2014. Contribution à l'étude phytochimique des extraits bruts des épices contenus dans le mélange Ras-elhanout. Mémoire master. Université Kasdi Merbah Ouargla, Algérie, p.99.

Brand-Williams W, Cuvelier ME, Berset C. 1995. Use of free radical method to evaluate antioxidant activity. Lebensm Wiss Technology, 25-30. https://doi.org/10.1016/S00236438(95)80008-5

Dohou N, Yamni K, Tahrouch S, Hassani L M I, Bodoc A et Gmira N. 2003. Screening phytochimique d'une endemique plante Ibero-marocain (Thymelaea lytroides), Bull. Soc. Pharm. Bordeaux, 61-78.

Emmanuel M. 2008. Aloe vera (l.) burm. f. Aspects pharmacologiques et clinique. Thèse de doctorat, Université de Nantes, Nantes, p. 224.

Enujiugha VN. 2010. The Antioxidant and Free Radical-Scavenging Capacity of Phenolics from African Locust Bean Seeds (Parkia biglobosa). Advances in Food Sciences, 32(2): 88-93.

Fangbo X, Yu Z, Mengqiu L, Qi C, Yonghong L, Xinmin L, Ruile P. 2015. Antioxidant and Anti-Fatigue Constituents of Okra. Nutrients, 7(10): 8846-8858. DOI: 10.3390/nu7105435.

Gbohaïda V, Agbangnan DCP, Nonviho G, Gnansounou M, Bothon FTD, Bogninou GSR, Avlessi F, Sohounhloué CKD. 2016. Chemical study and evaluation of the influence of Two physical parameters on polyphenols extraction from carapa procera leaves. World Journal of Pharmaceutical Research, 5(12): 108119.

Gbohaïda V, Mèdoatinsa SE, Nonviho G, Bogninou-Agbidinoukoun GSR, Agbangnan DCP et Sohounhloué DCK. 2015. Etude chimique et évaluation de l'Influence de la granulométrie sur la cinétique d'extraction des polyphénols naturels de Pterocarpus erinaceus acclimaté au Bénin, International Journal of Innovation and Applied Studies, 12(2): 325-333. 
Gisèle EL, Guy PN, Mariette K, Emmanuel MM, Siegfried DD. Evaluation des activités anti-inflammatoire et antiradicalaire de l'extrait au vin de palme des feuilles de Phragmanthera capitata (Sprengel) S. Balle (Loranthaceae) récoltées sur Psidium guajava au Cameroun, Int. J. Biol. Chem. Sci., 12(1): 233-243. DOI: https://dx.doi.org/10.4314/ijbcs.v12i1.18

Huang AG, Chen XH, Gao YZ, Che J.2007.Determination and analysis of ingredient in okra. Chin J Food Sci., 28: 451-455.

Itoua OYS, Elenga M, Moutsamboté JM, Mananga V, Mbemba F. 2015. Évaluation de la consommation et de la composition nutritionnelle des légumesfeuilles de Phytolacca dodecandra L'Herit consommés par les populations originaires des districts d'Owando et de Makoua. Journal of Animal \& Plant Sciences, 27(1): 4207-4218.

Kodjo SE, Kafui KO, SIMALOU, Pakoupati B, Amegnona A, Messanvi G. 2017. Etude comparative des activités antioxydantes d'extraits éthanoliques de feuilles, d'écorces et de racines de Cassia sieberiana, Int. J. Biol. Chem. Sci., 11(6): 2924-2935. DOI : https://dx.doi.org/10.4314/ijbcs.v11i6.29

Kouadio B, Djeneb C, Yao K, Guédé NZ. Potentiel antiradicalaire des extraits de feuilles de Bersama abyssinica Fresen. (Melianthaceae), Int. J. Biol. Chem. Sci., 11(6): 2962-2970. DOI: https://dx.doi.org/10.4314/ijbcs.v11i6.32

Kunli Wang Mo Li, Xin Wen, Xiaosong Chen, Zhengyu He, Yuanying Ni 2018. Optimization of ultrasound-assisted extraction of okra (Abelmoschus esculentus (L.) Moench) polysaccharides based on response surface methodology and antioxidant activity. International Journal of Biological Macromolecules, 114(15): 1056-1063.

Liao H, Dong W, Shi X, Liu H, Yuan K. 2012. Pharmacognosy Magazine, 8(30):
156-161. DOI: $\quad 10.4103 / 0973-$ 1296.96570.

Marius C, Gerard V, Antoine G. 1997. Le gombo, Abelmoschus esculentus (L.) Moench, une source possible de phospholipides. Agronomie et Biotechnologies. Oléagineux, Corps gras, Lipides, 4(5): 389-392.

N'Guessan K, Kadja B, Zirihi GN, Traore D et Ake-Assi L. 2009. Screening phytochimique de quelques plantes médicinales ivoiriennes utilisées en pays Krobou (Agboville, Côte-d'Ivoire). Sciences \& Nature, 6(1): 1-15.

Ouedraogo ZA. 2009. Caractérisation agromorphologique comparée de cinq variétés de Gombo (Abelmoschus esculentus (L.) Moench). Mémoire de fin de cycle en vue de l'obtention du diplôme d'ingénieur du développement rural, Université Polytechnique de BoboDioulasso, Burkina Faso, p. 61.

Serigne IMD, Alioune DF, Kady, Abdou S, Madieye S, Moussa S, Amadou M, William D, Emmanuel B. Evaluation de l'activité antioxydante des extraits hydro-ethanoliques des feuilles et écorces de Piliostigma thonningii Schumach, Int. J. Biol. Chem. Sci., 11(2): $\quad 768-776 . \quad$ DOI: https://dx.doi.org/10.4314/ijbcs.v11i2.19

Shankul K. 2014. Physicochemical, Phytochemical and toxicity studies on gum and mucilage from plant Abelmoschus esculentus. Journal of Phytopharmacology, 3(3): 200-203.

Soro TY, Traore F, Datte JY, Nene-Bi AS. 2009. Activité antipyrétique de l'extrait aqueux de Ximenia americana, Phytothérapie, 7(6): 297-303. DOI : 10.1007/s10298-009-0507-03

Traoré F. 2010. Proposition de formulation d'un sirop antipaludique à base de argemone mexicana L. (papaveraceae). Thèse de doctorat, Université de Bamako, Mali, p. 95. 